\title{
Saturation control of PVTOL aircraft on time scales
}

\author{
Özkan Öztürk ${ }^{a, *}$, Hacı M. Güzey ${ }^{b}$ \\ ${ }^{a}$ Department of Mathematics, Faculty of Arts and Sciences, Giresun University, Giresun, Turkey. \\ ${ }^{b}$ Electrical and Electronical Engineering, Erzurum Technical University, Yakutiye, Erzurum, Turkey.
}

\begin{abstract}
In this paper, we show the stability of a system for Planar Vertical Take-Off and Landing (PVTOL) aircraft on time scales by using a saturation function. This control technique on time scales is new and has successful results. Simulation results are also shown to validate our theoretical claims in some well-known time scales.
\end{abstract}

Keywords: Stability of aerial vehicles, stability on time scales, Lyapunov, PVTOL aircraft.

2020 MSC: 93D05, 93C10, 93C 95, 34N05.

(C)2020 All rights reserved.

\section{Introduction}

Flight control is a very important control problem that appears in many applications such as quadrotors, helicopters, spacecraft, aircraft and so on. Even if it is interesting to consider a simple aircraft, the complete dynamics of that aircraft might be very complex for the control purposes. In this article, we focus on PVTOL aircraft, shown in Figure 1, is the Bell X-22A VjSTOL and the last aircraft to be manufactured in New York, see [8]. There are several methods to control such a system in the literature, e.g., see $[9,13,14]$.

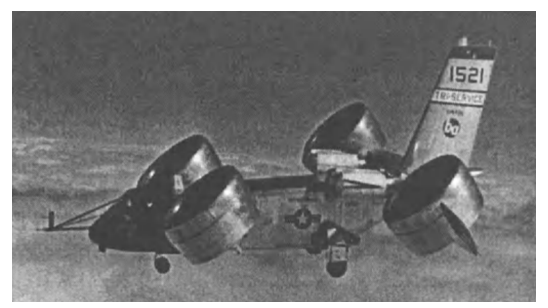

Figure 1: Bell X-22A VjSTOL.

\footnotetext{
*Corresponding author

Email addresses: ozturko@mst.edu (Özkan Öztürk), mehmet.guzey@erzurum.edu.tr (Hac1 M. Güzey)

doi: $10.22436 /$ jmcs.021.03.04
}

Received: 2019-02-23 Revised: 2020-02-07 Accepted: 2020-03-16 
A time scale, denoted by $\mathbb{T}$, is a nonempty closed subset of the real numbers, which was defined by Stefan Hilger in his PhD thesis in 1988, see [10]. His main purpose was to unify the results for differential and difference equations in one comprehensive theory and extend them for all other time scales. For example, when the domain is the set of real numbers $\mathbb{R}$, then the results are valid for ordinary differential equations and derivative is defined by $f^{\prime}(t)=\lim _{h \rightarrow 0} \frac{f(t+h)-f(t)}{h}$. When the domain is the set of integers $\mathbb{Z}$, then the results are valid for difference equations and derivative is defined by $\Delta f(t)=f(t+1)-f(t)$, where $\Delta$ is called difference operator. For general time scales, we use the notation $\mathrm{f}^{\Delta}$ and call it first delta-derivative of f. Besides $\mathbb{R}$ and $\mathbb{Z}$, some other well-known time scales are $q^{\mathbb{N}_{0}}=\left\{1, q, q^{2}, \cdots\right\}$ for $q>1$, $h \mathbb{Z}$ for $h>0$, $\mathbb{N}_{0}^{\frac{1}{2}}=\left\{n^{\frac{1}{2}} \quad n \in \mathbb{N}_{0}\right\}$ and so on. For more details, interested readers can read the books [3, 4] written by Bohner and Peterson in 2001 and 2003, respectively. In addition to that, for more information about the control theory and oscillation theory (which is very important for control), one can see [1, 17-19].

This article considers the following system

$$
\left\{\begin{array}{l}
x^{\Delta \Delta}(\mathrm{t})=-\mathrm{u}_{1}(\mathrm{t}) \sin \theta(\mathrm{t}) \\
\mathrm{y}^{\Delta \Delta}(\mathrm{t})=\mathrm{u}_{1}(\mathrm{t}) \cos \theta(\mathrm{t})-1, \\
\theta^{\Delta \Delta}(\mathrm{t})=\mathrm{u}_{2}(\mathrm{t})
\end{array}\right.
$$

where $x, y$ represent horizontal and vertical positions, and $\theta$ is the roll angle that the aircraft makes with the horizon and $t \in \mathbb{T}$. Here $f^{\Delta \Delta}$ represents the second order delta derivative of $f$. The control inputs $u_{1}$ and $\mathrm{u}_{2}$ are respectively the thrust (directed out the bottom of the aircraft) and the angular acceleration (the rolling moment) and -1 is the normalized gravitational acceleration. The control strategy for system (1.1) is the saturation function $S_{\varepsilon}: \mathbb{R} \rightarrow \mathbb{R}$ defined by

$$
S_{\varepsilon}(f(t))= \begin{cases}\varepsilon, & f(t)>\varepsilon \\ f(t), & -\varepsilon \leqslant f(t) \leqslant \varepsilon \\ -\varepsilon, & f(t)<-\varepsilon\end{cases}
$$

where $f: \mathbb{T} \rightarrow \mathbb{R}, \varepsilon>0$ and $t \in \mathbb{T}$. In many cases, saturation functions are used to limit the input signals to avoid damaging the actuators. When the input exceeds the limit, the output becomes constant.

This article is organized as follows. In Section 2, we give the basics of the time scale theory, which are used in the proofs of our main results. Section 3 shows our main results about the stability of system (1.1) and we provide simulation results for some certain time scales in Section 4. Finally, we have a conclusion in the last section.

\section{Time scale preliminaries}

\subsection{Basics of the Time Scale Theory}

In this section, basic definitions and theorems are given in order for readers to understand the time scale theory. For all these definitions and preliminaries, we refer [3] to readers.

Definition 2.1. For $t \in \mathbb{T}$, the forward jump operator $\sigma: \mathbb{T} \rightarrow \mathbb{T}$ is given by

$$
\sigma(t):=\inf \{s \in \mathbb{T}: \quad s>t\} \quad \text { for all } t \in \mathbb{T},
$$

while the backward jump operator $\rho: \mathbb{T} \rightarrow \mathbb{T}$ is defined by

$$
\rho(t):=\sup \{s \in \mathbb{T}: \quad s<t\} \quad \text { for all } t \in \mathbb{T} .
$$

Finally the graininess function $\mu: \mathbb{T} \rightarrow[0, \infty)$ is given by $\mu(t):=\sigma(t)-t$, for all $t \in \mathbb{T}$.

If sup $\mathbb{T}<\infty$, then $\mathbb{T}^{k}=\mathbb{T} \backslash(\rho(\sup \mathbb{T})$, sup $\mathbb{T}]$, and $\mathbb{T}^{k}=\mathbb{T}$ if sup $\mathbb{T}=\infty$. Suppose that $f: \mathbb{T} \rightarrow \mathbb{R}$ is a function. Then $f^{\sigma}: \mathbb{T} \rightarrow \mathbb{R}$ is defined by $f^{\sigma}(t)=f(\sigma(t))$ for all $t \in \mathbb{T}$. 
Definition 2.2. For any $\varepsilon$, if there exists a $\delta>0$ such that

$$
\left|f^{\sigma}(t)-f(s)-f^{\Delta}(t)(\sigma(t)-s)\right| \leqslant \varepsilon|\sigma(t)-s|
$$

for all $s \in(t-\delta, t+\delta) \cap \mathbb{T}$, then $f$ is called delta (or Hilger) differentiable on $\mathbb{T}^{\mathrm{k}}$ and $\mathrm{f}^{\Delta}$ is called delta derivative of $f$.

For the sake of the paper, when we say differentiable, we mean delta-differentiable throughout the whole article.

Theorem 2.3 ([3, Theorem 1.16]). Let $f: \mathbb{T} \rightarrow \mathbb{R}$ be a function with $\mathrm{t} \in \mathbb{T}^{\mathrm{k}}$. Then

a. if $f$ is differentiable at $\mathrm{t}, \mathrm{f}$ is continuous at $\mathrm{t}$;

$\mathrm{b}$. if $\mathrm{f}$ is continuous at $\mathrm{t}$ and $\mathrm{t}$ is right-scattered, then $\mathrm{f}$ is differentiable at $\mathrm{t}$ and

$$
f^{\Delta}(t)=\frac{f^{\sigma}(t)-f(t)}{\mu(t)}
$$

c. if $\mathrm{t}$ is right dense, then $\mathrm{f}$ is differentiable at $\mathrm{t}$ iff

$$
f^{\Delta}(t)=\lim _{s \rightarrow t} \frac{f(t)-f(s)}{t-s}
$$

exists as a finite number;

$\mathrm{d}$. if $\mathrm{f}$ is differentiable at $\mathrm{t}$, then

$$
f^{\sigma}(t)=f(t)+\mu(t) f^{\Delta}(t) .
$$

Equation (2.1) is called the simple useful formula and we use it on the Lyapunov function to show the stability of system (1.1).

Theorem 2.4 ([3, Theorem 1.20]). Let $\mathrm{f}, \mathrm{g}: \mathbb{T} \rightarrow \mathbb{R}$ be differentiable at $\mathrm{t} \in \mathbb{T}^{\mathrm{k}}$. Then

a. the sum $\mathrm{f}+\mathrm{g}: \mathbb{T} \rightarrow \mathbb{R}$ is differentiable at $\mathrm{t}$ with

$$
(f+g)^{\Delta}(t)=f^{\Delta}(t)+g^{\Delta}(t)
$$

b. if $\mathrm{fg}: \mathbb{T} \rightarrow \mathbb{R}$ is differentiable at $\mathrm{t}$, then

$$
(f g)^{\Delta}(t)=f^{\Delta}(t) g(t)+f^{\sigma}(t) g^{\Delta}(t)=f(t) g^{\Delta}(t)+f^{\Delta}(t) g^{\sigma}(t) .
$$

The following concepts must be introduced in order to define delta-integrable functions.

Definition 2.5 ([3, Definition 1.58]). $f: \mathbb{T} \rightarrow \mathbb{R}$ is called rd-continuous, if it is continuous at right dense points in $\mathbb{T}$ and its left sided limits exist as a finite number at left dense points in $\mathbb{T}$.

Theorem 2.6 ([3, Theorem 1.74]). Every rd-continuous function has an antiderivative. Moreover, F given by

$$
\mathrm{F}(\mathrm{t})=\int_{\mathrm{t}_{0}}^{\mathrm{t}} \mathrm{f}(\mathrm{s}) \Delta s \quad \text { for } \quad \mathrm{t} \in \mathbb{T}
$$

is called an antiderivative of $\mathrm{f}$.

Definition 2.7. A function $p: \mathbb{T} \rightarrow \mathbb{R}$ is called regressive provided that

$$
1+\mu(t) p(t) \neq 0 \quad \text { for all } t \in \mathbb{T}^{k} .
$$

The set of all regressive and rd-continuous functions is denoted by $\mathcal{R}=\mathcal{R}(\mathbb{T} ; \mathbb{R})$. The set $\mathcal{R}^{+}$of all positively regressive function is defined by

$$
\mathcal{R}^{+}=\left\{p \in \mathcal{R}: \quad 1+\mu(t) p(t)>0 \quad \text { for all } t \in \mathbb{T}^{\kappa}\right\} .
$$


Definition 2.8. If $p \in \mathcal{R}(\mathbb{T}, \mathbb{R})$, then the exponential function $e_{p}(t, s)$ on time scales is defined by

$$
e_{p}(t, s)=\exp \left(\int_{s}^{t} \Phi_{\mu(t)}(p(\tau)) \Delta \tau\right),
$$

where $\Phi_{h}(z)$ is the cylinder transformation given by

$$
\Phi_{h}(z)= \begin{cases}\frac{1}{h} \log (1+z h), & \text { if } h \neq 0, \\ z, & \text { if } h=0 .\end{cases}
$$

Particularly if $\mathbb{T}=\mathbb{R}$ then $e_{\lambda}(t, s)=e^{\lambda(t-s)}$ while $e_{\lambda}(t, s)=(1+\lambda)^{t-s}$ when $\mathbb{T}=\mathbb{Z}$ for a constant $\lambda \in \mathcal{R}$.

Now consider the system

$$
v^{\Delta}(\mathrm{t})=A v(\mathrm{t})
$$

where $A$ is real constant $n \times n$ matrix.

Definition 2.9 ([3, Definition 5.5]). An $n \times n$ matrix-valued function $A(t)$ on a time scale $\mathbb{T}$ is called regressive provided $I+\mu(t) A(t)$ is invertible for all $t \in \mathbb{T}^{k}$ and we denote the regressitivity by $A \in \mathcal{R}$.

The following lemma provides us a nice shortcut to determine the regresitivity of an $n \times n$ matrix $A$, see [3, Section 5.1]

Lemma 2.10. An $\mathrm{n} \times \mathrm{n}$ matrix-valued function $\mathrm{A}(\mathrm{t})$ is regressive if and only if the eigenvalues $\lambda_{i}(\mathrm{t})$ of $\mathrm{A}(\mathrm{t})$ are regressive for all $1 \leqslant i \leqslant n$, i.e., $1+\mu(t) \lambda_{i}(t) \neq 0$ for all $t \in \mathbb{T}^{k}$.

Finally, the following theorem shows us how to solve a linear dynamical system (2.2) on time scales in case that $A$ does not have $n$ linearly independent eigenvectors.

Theorem 2.11 ([3, Theorem 5.35 (Putzer Algorithm)]). Let $\mathrm{A} \in \mathcal{R}$ be a constant $\mathrm{n} \times \mathrm{n}$ matrix and $\mathrm{t}_{0} \in \mathbb{T}$. If $\lambda_{1}, \lambda_{2}, \ldots, \lambda_{n}$ are the eigenvalues of $A$, then

$$
e_{A}\left(t, t_{0}\right)=\sum_{i=0}^{n-1} r_{i+1}(t) P_{i},
$$

where $r(t)=\left[r_{1}(t), r_{2}(t), \cdots, r_{n}(t)\right]^{\top}$ is the solution of the initial value problem

$$
r^{\Delta}=\left[\begin{array}{ccccc}
\lambda_{1} & 0 & 0 & \cdots & 0 \\
1 & \lambda_{2} & 0 & \cdots & 0 \\
0 & 1 & \lambda_{3} & \cdots & 0 \\
\vdots & \ddots & \ddots & \ddots & \ddots \\
0 & \cdots & 0 & 1 & \lambda_{n}
\end{array}\right] r, \quad r\left(t_{0}\right)=\left[\begin{array}{c}
1 \\
0 \\
0 \\
\vdots \\
0
\end{array}\right]
$$

and $\mathrm{P}$-matrices $\mathrm{P}_{0}, \mathrm{P}_{1} \cdots, \mathrm{P}_{\mathrm{n}}$ are defined by $\mathrm{P}_{0}=\mathrm{I}$ and $\mathrm{P}_{\mathrm{k}+1}=\left(\mathrm{A}-\lambda_{\mathrm{k}+1} \mathrm{I}\right) \mathrm{P}_{\mathrm{k}}$ for $0 \leqslant \mathrm{k} \leqslant \mathrm{n}-1$.

\subsection{Stability theory on time scales}

This section provides us the basic definitions and theorems for Lyapunov and other type of stabilities. We use the conventions $\vec{z}=\vec{z}(t)$ and $\dot{V}(t, \vec{z})=[\mathrm{V}(\vec{z}(t))]^{\Delta}$ throughout. Here, $\mathrm{V}: \mathbb{R}^{n} \rightarrow[0, \infty)$ is called a Lyapunov function defined as

$$
\mathrm{V}(\vec{z})=\mathrm{V}_{1}\left(z_{1}\right)+\mathrm{V}_{2}\left(z_{2}\right)+\mathrm{V}_{3}\left(z_{3}\right)+\cdots,
$$

where each $V_{i}: \mathbb{R} \rightarrow \mathbb{R}$ is continuously differentiable. We use chain and product rules in order to compute $\dot{V}$. Even though the system is autonomous and $V=V(\vec{z}), \dot{V}$ depends on $t$ since the graininess function of $\mathbb{T}$ is not necessarily constant. In order for the readers to understand the notion of stability on time scales, we give the following definitions, see $[2,7,11,15,20]$. 
Definition 2.12. A function $\phi:[0, r] \rightarrow[0, \infty)$ is called a class of $\mathcal{K}$ if it is well-defined, continuous and strictly increasing on $[0, r]$ with $\phi(0)=0$.

Consider

$$
\vec{z}^{\Delta}=\mathrm{f}(\mathrm{t}, \vec{z}), \quad \vec{z}\left(\mathrm{t}_{0}\right)=\overrightarrow{z_{0}} \quad \mathrm{t} \geqslant \mathrm{t}_{0}, \quad \vec{z} \in \mathcal{D} \subset \mathbb{R}^{n},
$$

where $\mathcal{D}$ is a compact set. In addition, it is assumed that $f$ is continuous and that for system (2.3), the conditions are satisfied under which its solution $\vec{z}=\vec{z}\left(t, t_{0}, \overrightarrow{z_{0}}\right)$ exists and and is unique for all $t \geqslant t_{0} \in \mathbb{T}$. Further, we assume $f(t, \overrightarrow{0})=\overrightarrow{0} \in \mathcal{D}$ for all $t \in \mathbb{T}, t \geqslant t_{0}$ so that $\vec{z}=\overrightarrow{0}$ is a solution of equation (2.3).

Definition 2.13. The equilibrium solution $\vec{z}=\overrightarrow{0}$ of system (2.3) is called

(i) stable if for any $\varepsilon>0$ and $t_{0} \in \mathbb{T}$, there exists $\delta=\delta\left(\varepsilon, t_{0}\right)>0$ such that the condition $\left\|\overrightarrow{z_{0}}\right\|<\delta$ implies $\left\|\vec{z}\left(t, t_{0}, \overrightarrow{z_{0}}\right)\right\|<\varepsilon$ for all $t \in \mathbb{T}, t \geqslant t_{0}$;

(ii) attractive if

$$
\lim _{t \rightarrow \infty}\left\|\vec{z}\left(t, t_{0}, \overrightarrow{z_{0}}\right)\right\|=0
$$

holds for an initial condition $\overrightarrow{z_{0}} \in \mathbb{R}^{n}$;

(iii) asymptotically stable if it is stable and attractive;

(iv) exponentially stable if there exist constants $\alpha, \mathrm{d}>0$ with $-\alpha \in \mathcal{R}^{+}$and $\mathrm{K}>0$ such that the condition

$$
\| \vec{z}\left(t, t_{0}, \overrightarrow{z_{0}} \| \leqslant K\left(\left\|z_{0}\right\|, t_{0}\right)\left(e_{\ominus \alpha}\left(t, t_{0}\right)\right)^{\mathrm{d}}\right.
$$

holds for all $t \geqslant t_{0} \in \mathbb{T}$, where $\|\cdot\|$ denotes the Euclidean norm on $\mathbb{R}^{n}$, here $K$ and $\alpha$ are the so-called growth constants and $\ominus \alpha=\frac{-\alpha}{1+\mu(\mathrm{t}) \alpha}$;

(v) uniformly stable if for any $\varepsilon>0$ there exists a $\delta=\delta(\epsilon)>0$, independent of $t_{0}$, such that

$$
\left\|\overrightarrow{z_{0}}\right\|<\delta \Longrightarrow\left\|\vec{z}\left(t, t_{0}, \overrightarrow{z_{0}}\right)\right\|<\varepsilon, \quad t \in \mathbb{T}
$$

(vi) uniformly asymptotically stable if it is uniformly stable and there exists $c>0$, independent of $t_{0}$, such that

$$
\left\|\overrightarrow{z_{0}}\right\|<\mathrm{c} \Longrightarrow \lim _{\mathrm{t} \rightarrow \infty}\left\|\vec{z}\left(\mathrm{t}, \mathrm{t}_{0}, \overrightarrow{z_{0}}\right)\right\|=0
$$

uniformly in $t_{0}$;

(vii) uniformly exponentially stable if it is exponentially stable and constant $\mathrm{K}$ can be chosen independently of $\mathrm{t}_{0} \in \mathbb{T}$.

Notions of uniformity are only important for time-varying systems. Thus, for time-invariant systems, stability implies uniform stability and asymptotic stability implies uniform asymptotic stability. In other words, uniformity is a concept which guarantees that the equilibrium point is not losing stability, i.e., stability holds independently of $t_{0} \in \mathbb{T}$, see [16, Section 4.4] and [12, Section 4.5] to distinguish the definitions of stability and uniform stability.

Definition 2.14. A continuous function $P: \mathbb{R}^{n} \rightarrow \mathbb{R}$ with $P(\overrightarrow{0})=0$ is called

(i) positive definite (negative definite) on $\mathcal{D}$ if there exists a function $\phi \in \mathcal{K}$, such that $\phi(|\vec{z}|) \leqslant \mathrm{P}(\vec{z})(\phi(|\vec{z}|) \leqslant$ $-\mathrm{P}(\vec{z}))$ for $\vec{z} \in \mathcal{D}$;

(ii) positive semi-definite (negative semi-definite) on $\mathcal{D}$ if $\mathrm{P}(\vec{z}) \geqslant 0(\mathrm{P}(\vec{z}) \leqslant 0)$ for all $\vec{z} \in \mathcal{D}$.

Definition 2.15. A continuous function $Q:\left[t_{0}, \infty\right) \times \mathbb{R}^{n} \rightarrow \mathbb{R}$ with $Q(t, 0)=0$ is called

(i) positive definite (negative definite) on $\mathrm{t}_{0} \times \mathcal{D}$ if there exists a function $\phi \in \mathcal{K}$ such that $\phi(|\vec{z}|) \leqslant \mathrm{Q}(\mathrm{t}, \mathrm{x})$ $(\phi(|\vec{z}|) \leqslant-Q(t, x))$ for all $t \in \mathbb{T}, t \geqslant t_{0}$ and $\vec{z} \in \mathcal{D} ;$

(ii) positive semi-definite (negative semi-definite) on $\mathrm{t}_{0} \times \mathcal{D}$ if $\mathrm{Q}(\mathrm{t}, \vec{z}) \geqslant 0(\mathrm{Q}(\mathrm{t}, \mathrm{x}) \leqslant 0)$ for all $\mathrm{t} \in \mathbb{T}$, $\mathrm{t} \geqslant \mathrm{t}_{0}$ and $\vec{z} \in \mathcal{D}$. 
The following theorem gives the stability criteria for equation (2.3) on time scales.

Theorem 2.16 ([11, Theorem 1]). If there exists a continuously differentiable positive definite function $\mathrm{V}$ in a neighborhood of zero with $\dot{V}(t, x)$ negative semi-definite, then the equilibrium solution $\vec{z}=\overrightarrow{0}$ of equation (2.3) is stable.

The following lemma provides us a criteria for uniform exponential stability of a linear system depends only on the eigenvalues of its matrix, see [6, Theorem 3.2].

Lemma 2.17. Linear system (2.2) is uniformly exponentially stable if and only if $x^{\Delta}=\lambda x$ is uniformly exponentially stable for $\lambda \in \operatorname{spec}(A)$, where $\operatorname{spec}(A)$ denotes the set of eigenvalues of a matrix $A$.

Throughout the paper, we assume that sup $\mathbb{T}=\infty$ with a bounded graininess, i.e., $\limsup _{t \geqslant t_{0}} \mu(t)<$ $\infty$. Finally, the following lemma gives us the upper and lower exponential bounds for the circle minus exponential function, see [5].

Lemma 2.18. Suppose $\lim \sup \mu(t)<\infty$ and $\alpha \in \mathbb{R}^{+}$. Then

$$
e^{-\alpha(t-s)} \leqslant e_{\ominus \alpha}(t, s) \leqslant e_{-v}(t, s) \leqslant e^{-v(t-s)}
$$

for all $s, t \in \mathbb{T}$ with $\mathrm{t} \geqslant \mathrm{s}$, where $v \in \mathbb{R}^{+}$with $-v \in \mathbb{R}_{\mathrm{c}}^{+}(\mathbb{T}, \mathbb{R})\left(\mathbb{R}_{\mathrm{c}}^{+}(\mathbb{T}, \mathbb{R})\right.$ is the set of positively regressive constants.)

\section{Main results for stability}

In this section, we provide our main results for asymptotic stability of system (1.1). Before the main results, let us give the following lemma which helps us understand the procedure of the stability via the saturation function (1.2).

\subsection{Preliminary lemmas}

Since we are dealing with long integral solutions, for simplicity, let

$$
\left\{\begin{array}{l}
\mathrm{J}_{1}(\mathrm{t})=\int_{\mathrm{t}_{0}}^{\mathrm{t}} \frac{e_{-\mathrm{k}_{1} \ominus-\mathrm{k}_{2}}\left(\tau, \mathrm{t}_{0}\right)}{1-\mu(\tau) \mathrm{k}_{2}} \Delta \tau, \\
\mathrm{J}_{2}(\mathrm{t})=\int_{\mathrm{t}_{0}}^{\mathrm{t}} \frac{e_{-\mathrm{k}_{2} \ominus-\mathrm{k}_{3}}\left(\tau, \mathrm{t}_{0}\right)}{1-\mu(\tau) \mathrm{k}_{3}} \mathrm{~J}_{1}(\tau) \Delta \tau, \\
\mathrm{J}_{3}(\mathrm{t})=\int_{\mathrm{t}_{0}}^{\mathrm{t}} \frac{e_{-\mathrm{k}_{3} \ominus-\mathrm{k}_{4}}\left(\tau, \mathrm{t}_{0}\right)}{1-\mu(\tau) \mathrm{k}_{4}} \mathrm{~J}_{2}(\tau) \Delta \tau,
\end{array}\right.
$$

where $-k_{i} \in \mathcal{R}^{+}, k_{1} \geqslant k_{2} \geqslant k_{3} \geqslant k_{4}>0$ for $i=1,2,3,4$ and $(p \ominus q)(t)=\frac{p(t)-q(t)}{1+\mu(t) q(t)}, t \in \mathbb{T}^{k}$. Throughout the article, we assume that the integrals $J_{1}(t), J_{2}(t)$ and $J_{3}(t)$ have finite limits as $t \rightarrow \infty$. We use these integrals for the solution of the system in the following lemma.

Lemma 3.1. Consider the following system

$$
\left\{\begin{array}{l}
\xi_{1}^{\Delta}(t)=k_{2} \xi_{2}(t)+k_{3} \xi_{3}(t)+k_{4} \xi_{4}(t)+u(t), \\
\xi_{2}^{\Delta}(t)=k_{3} \xi_{3}(t)+k_{4} \xi_{4}(t)+u(t) \\
\xi_{3}^{\Delta}(t)=k_{4} \xi_{4}(t)+u(t) \\
\xi_{4}^{\Delta}(t)=u(t)
\end{array}\right.
$$

with the control input

$$
u(t)=-k_{4} S_{\frac{\varepsilon}{k_{4}}}\left(\xi_{4}\right)-k_{3} S_{\frac{\varepsilon}{2 k_{3}}}\left(\xi_{3}\right)-k_{2} S_{\frac{\varepsilon}{4 k_{2}}}\left(\xi_{2}\right)-k_{1} S_{\frac{\varepsilon}{8 k_{1}}}\left(\xi_{1}\right),
$$

where $S$ is defined by (1.2), $k_{i}, \varepsilon>0$ and $-k_{i} \in \mathcal{R}^{+}$for $i=1,2,3,4$. Suppose also that $\mu(t) \leqslant k$ and $t \geqslant t_{0}$, where 
$\mathrm{k}=\min \left\{\frac{16}{225 \mathrm{k}_{4}}, \frac{8}{49 \mathrm{k}_{3}}, \frac{4}{9 \mathrm{k}_{2}}, \frac{9}{10 \mathrm{k}_{1}}\right\}$. Then system (3.1) with (3.2) is uniformly exponentially stable and therefore uniformly asymptotically stable.

Proof. First note that there is no singular solution of the second kind, i.e., there does not exist a finite $\mathrm{T} \geqslant \mathrm{t}_{0}$ such that

$$
\lim _{t \rightarrow T^{-}} \xi_{i}(t)=\infty
$$

for $T, t_{0} \in \mathbb{T}$ since the control law (3.2) is bounded. Now, we start from the last equation of system (3.1). By plugging (3.2) into the last equation of system (3.1), we have

$$
\xi_{4}^{\Delta}(t)=-k_{4} S_{\frac{\varepsilon}{k_{4}}}\left(\xi_{4}(t)\right)-k_{3} S_{\frac{\varepsilon}{2 k_{3}}}\left(\xi_{3}(t)\right)-k_{2} S_{\frac{\varepsilon}{4 k_{2}}}\left(\xi_{2}(t)\right)-k_{1} S_{\frac{\varepsilon}{8 k_{1}}}\left(\xi_{1}(t)\right) .
$$

Define a Lyapunov function $V_{4}\left(t, \xi_{4}\right)=\xi_{4}^{2}(t)$ for $t \in \mathbb{T}$. Then by using the product rule on time scales and the simple useful formula for all $\left|\xi_{4}(t)\right| \geqslant \frac{\varepsilon}{k_{4}}$, we get

$$
\begin{aligned}
\dot{V}_{4} & =\xi_{4}^{\Delta}(t)\left(\xi_{4}^{\sigma}(t)+\xi_{4}(t)\right)=2 \xi_{4}^{\Delta}(t) \xi_{4}(t)+\mu(t)\left(\xi_{4}^{\Delta}(t)\right)^{2} \\
& \leqslant 2\left(-\varepsilon+\frac{\varepsilon}{2}+\frac{\varepsilon}{4}+\frac{\varepsilon}{8}\right)\left|\xi_{4}(t)\right|+\mu(t)\left(\varepsilon+\frac{\varepsilon}{2}+\frac{\varepsilon}{4}+\frac{\varepsilon}{8}\right)^{2}
\end{aligned}
$$

because $k_{4} S_{\frac{\varepsilon}{k_{4}}}\left(\xi_{4}(t)\right) \cdot \xi_{4}(t)=\varepsilon\left|\xi_{4}(t)\right|$ for $t \in \mathbb{T}$. Hence, since for $\left|\xi_{4}(t)\right| \geqslant \frac{\varepsilon}{k_{4}}$ and $\mu(t) \leqslant \frac{16}{225 k_{4}}, t \in \mathbb{T}$, we have

$$
\dot{V}_{4} \leqslant-\frac{\varepsilon}{4}\left|\xi_{4}(t)\right|+\mu(t) \frac{225}{64} \varepsilon^{2} \leqslant-\varepsilon^{2}\left(\frac{1}{4 k_{4}}-\mu(t) \frac{225}{64}\right) \leqslant 0 .
$$

It follows that $V$ is negative semi definite for $\left|\xi_{4}(t)\right| \geqslant \frac{\varepsilon}{k_{4}}$ and $\mu(t) \leqslant \frac{16}{225 k_{4}}, t \in \mathbb{T}$. Therefore, there exists a $t_{1} \geqslant t_{0}$ such that $\left|\xi_{4}(t)\right|$ is bounded for all $t \geqslant t_{1}$, i.e. $\left|\xi_{4}(t)\right| \leqslant \frac{\varepsilon}{k_{4}}$. Then the controller $u$ turns out to be

$$
u(t)=-k_{4} \xi_{4}(t)-k_{3} S_{\frac{\varepsilon}{2 k_{3}}}\left(\xi_{3}(t)\right)-k_{2} S_{\frac{\varepsilon}{4 k_{2}}}\left(\xi_{2}(t)\right)-k_{1} S_{\frac{\varepsilon}{8 k_{1}}}\left(\xi_{1}(t)\right)
$$

for all $t \geqslant t_{1}$ on time scales. Now by plugging (3.3) into the third equation of (3.1), we have

$$
\xi_{3}^{\Delta}(t)=-k_{3} S_{\frac{\varepsilon}{2 k_{3}}}\left(\xi_{3}(t)\right)-k_{2} S_{\frac{\varepsilon}{4 k_{2}}}\left(\xi_{2}(t)\right)-k_{1} S_{\frac{\varepsilon}{8 k_{1}}}\left(\xi_{1}(t)\right) .
$$

Define a Lyapunov function $V_{3}$ as

$$
V_{3}\left(t, \xi_{3}\right)=\xi_{3}^{2}(t), \quad t \geqslant t_{1}
$$

With the same procedure, we have

$$
\dot{V}_{3}\left(t, \xi_{3}\right) \leqslant-\varepsilon^{2}\left(\frac{1}{8 k_{3}}-\frac{49}{64} \mu(t)\right) \leqslant 0
$$

for $\left|\xi_{3}(t)\right| \geqslant \frac{\varepsilon}{2 k_{3}}$ and $\mu(t) \leqslant \frac{8}{49 k_{3}}, t \geqslant t_{1}$, i.e. $V_{3}$ is negative semi definite. Therefore, there exists a $t_{2} \geqslant t_{1}$ such that $\left|\xi_{3}(t)\right| \leqslant \frac{\varepsilon}{2 k_{3}}$ for $t \geqslant t_{2}$. Then the control input $u$ and the second equation of system (3.1) end up with

$$
u(t)=-k_{4} \xi_{4}(t)-k_{3} \xi_{3}(t)-k_{2} S_{\frac{\varepsilon}{4 k_{2}}}\left(\xi_{2}(t)\right)-k_{1} S_{\frac{\varepsilon}{8 k_{1}}}\left(\xi_{1}(t)\right)
$$

and

$$
\xi_{2}^{\Delta}(t)=-k_{2} S_{\frac{\varepsilon}{4 k_{2}}}\left(\xi_{2}\right)-k_{1} S_{\frac{\varepsilon}{8 k_{1}}}\left(\xi_{1}\right),
$$

respectively for $t \geqslant t_{2} \in \mathbb{T}$. Continuing with a similar procedure yields us that there exists a large $t_{3} \in \mathbb{T}$ for $t_{3} \geqslant t_{2} \geqslant t_{1}$ such that $\left|\xi_{4}(t)\right| \leqslant \frac{\varepsilon}{k_{4}},\left|\xi_{3}(t)\right| \leqslant \frac{\varepsilon}{2 k_{3}},\left|\xi_{2}(t)\right| \leqslant \frac{\varepsilon}{4 k_{2}}$ and $\left|\xi_{1}(t)\right| \leqslant \frac{\varepsilon}{8 k_{1}}$ for $\mu(t) \leqslant k$ and $t \geqslant t_{3}$, where $k=\min \left\{\frac{16}{225 k_{4}}, \frac{8}{49 k_{3}}, \frac{4}{9 k_{2}}, \frac{9}{10 k_{1}}\right\}$. Hence, the control input $u$ finally ends up with

$$
u(t)=-k_{4} \xi_{4}-k_{3} \xi_{3}-k_{2} \xi_{2}-k_{1} \xi_{1}
$$


for $t \geqslant t_{3}$ and $\mu(t) \leqslant k$. By using the updated control input (3.4), we have that system (3.1) turns out to be

$$
\xi^{\Delta}(t)=A \xi(t),
$$

where $\xi=\left[\begin{array}{llll}\xi_{1} & \xi_{2} & \xi_{3} & \xi_{4}\end{array}\right]^{\top}$ and

$$
A=\left[\begin{array}{cccc}
-k_{1} & 0 & 0 & 0 \\
-k_{1} & -k_{2} & 0 & 0 \\
-k_{1} & -k_{2} & -k_{3} & 0 \\
-k_{1} & -k_{2} & -k_{3} & -k_{4}
\end{array}\right]
$$

for $\mu(t) \leqslant k, t \geqslant t_{3}$. System (3.5) is a simple linear dynamical system and can be solved by using Theorem 2.11 since the eigenvalues $\lambda_{i}=\left\{-k_{1},-k_{2},-k_{3},-k_{4}\right\}$ of $A$ satisfies $1-\mu(t) k_{i}>0$ for all $i=1,2,3,4$, i.e., $A \in \mathcal{R}^{+}$. Therefore, the general solution of system (3.5) is

$$
\xi(t)=e_{A}\left(t, t_{0}\right) \xi_{0}=\left[e_{-k_{1}}\left(t, t_{0}\right) P_{0}+e_{-k_{2}}\left(t, t_{0}\right) J_{1}(t) P_{1}+e_{-k_{3}}\left(t, t_{0}\right) J_{2}(t) P_{2}+e_{-k_{4}}\left(t, t_{0}\right) J_{3}(t) P_{3}\right] \xi_{0},
$$

where $\xi_{0}$ is any arbitrary constant vector, $P_{0}=I_{4 \times 4}$, and $P_{1}$ through $P_{3}$ are constant matrices that can easily be calculated by the formula given in Theorem 2.11. Then by Lemma 2.18 and the definition of the uniform exponential stability, we have that system (3.1) with the controller (3.2) is uniformly exponentially stable. In addition to that, by Theorem 2.2.5 in [15], it follows that system (3.1) is uniformly asymptotically stable. This completes the proof.

Now, by using Lemma 3.1, we obtain the following lemma, which proves the uniform asymptotic stability of chain of integrators of four dimension.

Lemma 3.2. Consider the chain of integrators

$$
\left\{\begin{array}{l}
z_{1}^{\Delta}(\mathrm{t})=\mathrm{k}_{2} z_{2}(\mathrm{t}) \\
z_{2}^{\Delta}(\mathrm{t})=\mathrm{k}_{3} z_{3}(\mathrm{t}) \\
z_{3}^{\Delta}(\mathrm{t})=\mathrm{k}_{4} z_{4}(\mathrm{t}) \\
z_{4}^{\Delta}(\mathrm{t})=\mathrm{u}(\mathrm{t})
\end{array}\right.
$$

with the control input

$$
\begin{aligned}
u(t)= & -k_{4} S_{\frac{\varepsilon}{k_{4}}}\left(z_{4}(t)\right)-k_{3} S_{\frac{\varepsilon}{2 k_{3}}}\left(z_{4}(t)+z_{3}(t)\right)-k_{2} S_{\frac{\varepsilon}{4 k_{2}}}\left(z_{4}(t)+\left(1+\frac{k_{3}}{k_{4}}\right) z_{3}(t)+z_{2}(t)\right) \\
& -k_{1} S_{\frac{\varepsilon}{8 k_{1}}}\left[z_{4}(t)+\left(1+\frac{k_{3}}{k_{4}}+\frac{k_{2}}{k_{4}}\right) z_{3}(t)+\left(1+\frac{k_{2}}{k_{3}}+\frac{k_{2}}{k_{4}}\right) z_{2}(t)+z_{1}(t)\right],
\end{aligned}
$$

where $S$ is defined by (1.2), $-k_{i} \in \mathcal{R}^{+}$, and $k_{1} \geqslant k_{2} \geqslant k_{3} \geqslant k_{4}>0$ for $i=1,2,3,4$. Then system (3.6) with the controller (3.7) is uniformly asymptotically stable.

Proof. Consider the following transformation:

$$
\left\{\begin{array}{l}
\xi_{1}(t)=z_{4}(t)+\left(1+\frac{k_{3}}{k_{4}}+\frac{k_{2}}{k_{4}}\right) z_{3}(t)+\left(1+\frac{k_{2}}{k_{3}}+\frac{k_{2}}{k_{4}}\right) z_{2}(t)+z_{1}(t) \\
\xi_{2}(t)=z_{4}(t)+\left(1+\frac{k_{3}}{k_{4}}\right) z_{3}(t)+z_{2}(t) \\
\xi_{3}(t)=z_{4}(t)+z_{3}(t) \\
\xi_{4}(t)=z_{4}(t)
\end{array}\right.
$$

Then, we have that system (3.6) is transformed into the following system:

$$
\left\{\begin{array}{l}
\xi_{1}^{\Delta}(t)=k_{2} \xi_{2}(t)+k_{3} \xi_{3}(t)+k_{4} \xi_{4}(t)+u(t), \\
\xi_{2}^{\Delta}(t)=k_{3} \xi_{3}(t)+k_{4} \xi_{4}(t)+u(t) \\
\xi_{3}^{\Delta}(t)=k_{4} \xi_{4}(t)+u(t) \\
\xi_{4}^{\Delta}(t)=u(t)
\end{array}\right.
$$


where controller (3.7) is also turned into

$$
u(t)=-k_{4} S_{\frac{\varepsilon}{k_{4}}}\left(\xi_{4}(t)\right)-k_{3} S_{\frac{\varepsilon}{2 k_{3}}}\left(\xi_{3}(t)\right)-k_{2} S_{\frac{\varepsilon}{4 k_{2}}}\left(\xi_{2}(t)\right)-k_{1} S_{\frac{\varepsilon}{8 k_{1}}}\left(\xi_{1}(t)\right) .
$$

Therefore, applying Lemma 3.1 for system (3.8) with (3.9), the assertion follows.

In the next two subsections, we show the asymptotic stability of system (1.1) by dividing it into subsystems and then each subsystem is shown to be asymptotically stable. In other words, we first control the vertical position $y$ and make it stable. Then, we deal with the stability of the roll angle and horizontal position by using the lemmas provided in the previous sections .

\subsection{Stability of the vertical position}

This section shows us the asymptotic stability of the subsystem $y$ of system (1.1). Please note that the controller $v_{1}$ is not given in the theorem, it is determined in the proof by using the same process as in Lemma 3.2.

Theorem 3.3. Consider the subsystem

$$
y^{\Delta \Delta}(t)=u_{1}(t) \cos \theta(t)-1
$$

with the controller

$$
\mathrm{u}_{1}(\mathrm{t})=\frac{v_{1}(\mathrm{t})+1}{\cos \left(\mathrm{S}_{\frac{\pi}{2}}(\theta(\mathrm{t}))\right)}
$$

where $S$ is the saturation function and $v_{1}$ is to be determined later. Then system (3.10) is uniformly asymptotically stable.

Proof. Let us consider subsystem (3.10) and the controller input (3.11). Here, we assume that there exists a finite $T$ such that $|\theta(t)| \leqslant 1<\frac{\pi}{2}$ for $t \geqslant T$ (to be proved next section). Then, by using controller (3.11), we have $y^{\Delta \Delta}=v_{1}(t)$ and rewriting this as a system gives us:

$$
\left\{\begin{array}{l}
y_{1}^{\Delta}(t)=c_{2} y_{2}(t), \\
y_{2}^{\Delta}(t)=v_{1}(t),
\end{array}\right.
$$

where $y_{1}=c_{2} y$ and $y_{2}=y^{\Delta}$ for positive constant $c_{2}$ and $-c_{2} \in \mathcal{R}^{+}$. Now by using the same argument as in Lemma 3.2, we can determine the controller $v_{1}$ as

$$
v_{1}(t)=-c_{2} S_{\frac{1}{2 c_{2}}}\left(y_{2}(t)\right)-c_{1} S_{\frac{1}{4 c_{1}}}\left(y_{1}(t)+y_{2}(t)\right)
$$

for $c_{1} \geqslant c_{2}>0$, and $-c_{1} \in \mathcal{R}^{+}$for $t \geqslant T$, which implies that system (3.12) is uniformly asymptotically stable. Therefore, the assertion follows.

\subsection{Stability of the roll angle and horizontal position}

In this section, we show the asymptotic stability of the subsystem including the roll angle $\theta$ and the horizontal replacement $x$. Therefore, consider the subsystem

$$
\left\{\begin{array}{l}
x^{\Delta \Delta}(\mathrm{t})=-\mathrm{u}_{1}(\mathrm{t}) \sin \theta(\mathrm{t}) \\
\theta^{\Delta \Delta}(\mathrm{t})=\mathrm{u}_{2}(\mathrm{t})
\end{array}\right.
$$

Plugging controller (3.11) into subsystem (3.14) yields us

$$
\left\{\begin{array}{l}
x^{\Delta \Delta}(t)=-\left(v_{1}(t)+1\right) \tan \theta(t), \\
\theta^{\Delta \Delta}(t)=u_{2}(t),
\end{array}\right.
$$


where $v_{1}$ is defined by (3.13). Next, we take subsystem (3.15) into account with the controllers $v_{1}$ and $u_{2}$ and determine the stability of the system by using stability theorems given in Section 2.2 and lemma in Section 3.1. Note that system (3.15) is a perturbed time scale system, so we give the following lemma to work on stability for such systems on time scales.

Lemma 3.4 ([2, Theorem 3.5]). Suppose that the equilibrium point $\beta=0$ of system $\beta^{\Delta}(t)=A(t) \beta$ is uniformly exponentially stable with growth constants $\mathrm{K}$ and $\alpha$. Suppose also that the perturbing term $\mathrm{F}$ of

$$
\beta^{\Delta}(t)=A(t) \beta+F(t, \beta),
$$

where $\mathrm{F}(\mathrm{t}, 0)=0$, satisfies the growth rate condition

$$
\|F(t, \beta)\| \leqslant P(t,\|\beta\|), \quad t \in \mathbb{T}, \quad \beta \in \mathbb{R}^{n},
$$

where $\mathrm{P}, \mathrm{Q}: \mathbb{T} \times \mathbb{R}^{+} \rightarrow \mathbb{R}^{+}$are rd-continuous functions satisfying

$$
0 \leqslant P(t, \beta)-P(t, \zeta) \leqslant Q(t, \zeta)(\beta-\zeta), t \in \mathbb{T}, \beta \geqslant \zeta \geqslant 0,
$$

where $\mathrm{P}(\mathrm{t}, 0)=0$ for all $\mathrm{t} \in \mathbb{T}$ and

$$
\int_{\mathrm{t}_{0}}^{\infty} \frac{\mathrm{Q}(\mathrm{s}, \mathrm{m})}{1-\alpha \mu(\mathrm{s})} \Delta \mathrm{s}<\infty
$$

for all $\left(t_{0}, m\right) \in \mathbb{T} \times\left[0, m_{0}\right]$, where $m_{0}>0$. Then the equilibrium point $\beta=0$ of perturbed system (3.16) is uniformly asymptotically stable.

Next, we use Lemma 3.4 to show the stability of a specific perturbed system, which is used for the following results.

Theorem 3.5. The perturbed system $\xi^{\Delta}(t)=A \xi(t)+f(t, \xi)$ is uniformly asymptotically stable, where

$$
\begin{aligned}
A & =\left[\begin{array}{cccc}
-1 & 0 & 0 & 0 \\
-1 & -1 & 0 & 0 \\
-1 & -1 & -1 & 0 \\
-1 & -1 & -1 & -1
\end{array}\right], \\
f(t, \xi) & =\left[\begin{array}{c}
3 v_{1}(t) \tan \left(\xi_{3}-\xi_{4}\right)(t)+3\left(\tan \left(\xi_{3}-\xi_{4}\right)(t)-\left(\xi_{3}-\xi_{4}\right)(t)\right) \\
v_{1}(t) \tan \left(\xi_{3}-\xi_{4}\right)(t)+\left(\tan \left(\xi_{3}-\xi_{4}\right)(t)-\left(\xi_{3}-\xi_{4}\right)(t)\right) \\
0 \\
0
\end{array}\right]
\end{aligned}
$$

for $\left|\left(\xi_{3}-\xi_{4}\right)(t)\right| \leqslant \frac{1}{8}, t \in \mathbb{T}$ and $A \in \mathcal{R}^{+}$.

Proof. First note that the matrix $A$ is the special case of the matrix $A$ in Lemma 3.1, i.e., $k_{i}=1, i=$ $1,2,3,4$ and we have already shown that non-perturbed system $\xi^{\Delta}(t)=A \xi(t)$ is uniformly exponentially/asymptotically stable. Therefore, we only show $\xi^{\Delta}(t)=A \xi(t)+f(t, \xi)$ is also uniformly asymptotically stable. To do this, we use Lemma 3.4. First note that

$$
\left|\tan \left(\xi_{3}-\xi_{4}\right)(t)-\left(\xi_{3}-\xi_{4}\right)(t)\right| \leqslant \frac{\left(\xi_{3}-\xi_{4}\right)^{2}(t)}{6}
$$

for $\left(\xi_{3}-\xi_{4}\right)(t) \in\left[-\frac{1}{8}, \frac{1}{8}\right], t \in \mathbb{T}$. Also, since $y(t)$ is uniformly asymptotically stable, we can choose a large finite time $T \in \mathbb{T}$ such that $\left|v_{1}(t)\right| \leqslant \frac{1}{84}$ for $t \geqslant T, t \in \mathbb{T}$. Then

$$
\|f(t, \xi)\|^{2} \leqslant\left(\frac{5}{5292}+\frac{10}{36}+\frac{20}{3024}\right)\left(\xi_{3}-\xi_{4}\right)^{4}+\left(\frac{5}{5292}+\frac{20}{4032}\right)\left(\xi_{3}-\xi_{4}\right)^{2}
$$




$$
\leqslant \mathrm{L}\left(\left|\xi_{3}\right|+\left|\xi_{4}\right|\right)^{2} \leqslant 4 \mathrm{~L}\left(\left|\xi_{1}\right|^{2}+\left|\xi_{2}\right|^{2}+\left|\xi_{3}\right|^{2}+\left|\xi_{4}\right|^{2}\right)=\mathrm{P}(\mathrm{t},\|\xi\|)
$$

for $L \approx 0.291$ and $\left|v_{1}(t)\right| \leqslant \frac{1}{84}, t \geqslant T$. Note also that condition (3.17) is satisfied for $Q \geqslant 1$, say $Q(t, \zeta)=$ 2. Observe also that condition (3.18) is satisfied because $J_{1}(\infty)<\infty$ for $k_{1}=k_{2}=1$. Therefore, by Lemma 3.4, we have that our perturbed system is uniformly asymptotically stable. This completes the assertion.

Finally, the following last theorem shows us the stability of the roll angle $\theta$ and horizontal position $x$ for system (1.1).

Theorem 3.6. System (3.15) with control input

$$
\mathrm{u}_{2}(\mathrm{t})=\mathrm{S}_{\frac{1}{12}}\left(-\theta^{\Delta}(\mathrm{t})\right)+\mathrm{S}_{\frac{1}{24}}\left(\left(-\theta-\theta^{\Delta}\right)(\mathrm{t})\right)+\mathrm{S}_{\frac{1}{48}}\left(\left(x^{\Delta}-2 \theta-\theta^{\Delta}\right)(\mathrm{t})\right)+\mathrm{S}_{\frac{1}{96}}\left(\left(x+3 x^{\Delta}-3 \theta-\theta^{\Delta}\right)(\mathrm{t})\right)
$$

is uniformly asymptotically stable.

Proof. Consider system (3.15) with the controller (3.19). Making the change of variable $\gamma=-\theta$ and $v_{2}=-\mathrm{u}_{2}$ for all $\mathrm{t} \in \mathbb{T}$ transforms system (3.15) into

$$
\left\{\begin{array}{l}
x^{\Delta \Delta}(\mathrm{t})=\gamma(\mathrm{t})+(\tan \gamma-\gamma)(\mathrm{t})+v_{1}(\mathrm{t}) \tan \gamma(\mathrm{t}) \\
\gamma^{\Delta \Delta}(\mathrm{t})=v_{2}(\mathrm{t})
\end{array}\right.
$$

Rewriting system (3.20) as a system of first order dynamic equations on time scales gives:

$$
\left\{\begin{array}{l}
x_{1}^{\Delta}(t)=x_{2}(t) \\
x_{2}^{\Delta}(t)=\gamma_{1}(t)+\left(\tan \gamma_{1}-\gamma_{1}\right)(t)+v_{1}(t) \tan \gamma_{1}(t) \\
\gamma_{1}^{\Delta}(t)=\gamma_{2}(t) \\
\gamma_{2}^{\Delta}(t)=v_{2}(t)
\end{array}\right.
$$

Then by the following transformation

$$
\left\{\begin{array}{l}
\xi_{1}(t)=x_{1}(t)+3 x_{2}(t)+3 \gamma_{1}(t)+\gamma_{2}(t) \\
\xi_{2}(t)=x_{2}(t)+2 \gamma_{1}(t)+\gamma_{2}(t) \\
\xi_{3}(t)=\gamma_{1}(t)+\gamma_{2}(t) \\
\xi_{4}(t)=\gamma_{2}(t)
\end{array}\right.
$$

for all $t \in \mathbb{T}$, we have:

$$
\left\{\begin{array}{l}
\xi_{1}^{\Delta}(t)=\xi_{2}+\xi_{3}+\xi_{4}+v_{2}+3 v_{1} \tan \left(\xi_{3}-\xi_{4}\right)+3\left(\tan \left(\xi_{3}-\xi_{4}\right)-\left(\xi_{3}-\xi_{4}\right)\right), \\
\xi_{2}^{\Delta}(t)=\xi_{3}+\xi_{4}+v_{2}+v_{1} \tan \left(\xi_{3}-\xi_{4}\right)+\left(\tan \left(\xi_{3}-\xi_{4}\right)-\left(\xi_{3}-\xi_{4}\right)\right) \\
\xi_{3}^{\Delta}(t)=\xi_{4}+v_{2} \\
\xi_{4}^{\Delta}(t)=v_{2}
\end{array}\right.
$$

Note that system (3.21) is very similar to system (3.1) but there is an additional perturbed term. We complete the proof with two claims. First, we claim that solutions of system (3.21) enter a bounded region after a finite time. And in the second claim, we prove that system (3.21) is uniformly asymptotically stable in that region region.

Claim 1: We claim that using the control input $v_{2}=-S_{\frac{1}{12}}\left(\xi_{4}(t)\right)-S_{\frac{1}{24}}\left(\xi_{3}(t)\right)-S_{\frac{1}{48}}\left(\xi_{2}(t)\right)-S_{\frac{1}{96}}\left(\xi_{1}(t)\right)$ gives us that the solutions of system (3.21) enter a bounded region $X$ defined by

$$
x=\left\{\left(\xi_{1}, \xi_{2}, \xi_{3}, \xi_{4}\right):\left|\xi_{1}(t)\right| \leqslant \frac{1}{96},\left|\xi_{2}(t)\right| \leqslant \frac{1}{48},\left|\xi_{3}(t)\right| \leqslant \frac{1}{24},\left|\xi_{4}(t)\right| \leqslant \frac{1}{12}, t \in \mathbb{T}\right\}
$$

after a finite time on $\mathbb{T}$. 
Proof of Claim 1: By a similar discussion as in Lemma 3.1, we can have a large $T_{1} \in \mathbb{T}$ such that $\left|\xi_{3}(t)\right| \leqslant \frac{1}{24}$ and $\left|\xi_{4}(t)\right| \leqslant \frac{1}{12}$ for $t \geqslant T_{1}$. Now, let us show that the other component functions $\xi_{1}$ and $\xi_{2}$ stay bounded after a finite time. First note that $\left|-\gamma_{1}\right|=|-\gamma|=|\theta|=\left|\xi_{4}-\xi_{3}\right| \leqslant \frac{1}{8}$, i.e., $|\theta|$ is bounded for $T_{1} \geqslant t$. Therefore, $\left|\tan \left(\xi_{3}-\xi_{4}\right)-\left(\xi_{3}-\xi_{4}\right)\right| \leqslant \frac{66}{10^{5}}$ and $\left|\tan \left(\xi_{3}-\xi_{4}\right)\right| \leqslant \frac{12}{100}$ for $t \geqslant T_{1}$. In addition to that, we can choose an upper bound for $v_{1}(t)$ for $t \geqslant T_{1}$ because $y(t)$ is asymptotically stable. Therefore, one can have $\left|v_{1}(t)\right| \leqslant \frac{1}{84}$ for $t \geqslant T_{1}$. In the light of all this information, now let us prove that $\xi_{2}$ and $\xi_{1}$ are also bounded after a finite time. Since $\xi_{3}$ and $\xi_{4}$ are bounded by $\frac{1}{24}$ and $\frac{1}{12}$ for $t \geqslant T_{1}$, we have that the control input $v_{2}$ turns out to be

$$
v_{2}(t)=-\xi_{4}-\xi_{3}-S_{\frac{1}{48}}\left(\xi_{2}\right)-S_{\frac{1}{96}}\left(\xi_{1}\right)
$$

for $t \geqslant T_{1}$ by the definition of the saturation function. Then the second equation of system (3.21) becomes

$$
\xi_{2}^{\Delta}(t)=-S_{\frac{1}{48}}\left(\xi_{2}\right)-S_{\frac{1}{96}}\left(\xi_{1}\right)+v_{1} \tan \left(\xi_{3}-\xi_{4}\right)+\left(\tan \left(\xi_{3}-\xi_{4}\right)-\left(\xi_{3}-\xi_{4}\right)\right)
$$

for $t \geqslant T_{1} \in \mathbb{T}$. Hence, set a Lyapunov function $V_{2}\left(t, \xi_{2}\right)=\xi_{2}^{2}(t)$ for $t \geqslant T_{1}$. Then by the product rule and the simple useful formula (2.1) on time scales, we have

$$
\begin{aligned}
\dot{V}_{2}\left(t, \xi_{2}\right)= & \xi_{2}^{\Delta}\left(2 \xi_{2}+\mu(t) \xi_{2}^{\Delta}\right), \\
\leqslant & 2\left[\frac{-1}{48}+\frac{1}{96}+v_{1} \tan \left(\xi_{3}-\xi_{4}\right)+\left(\tan \left(\xi_{3}-\xi_{4}\right)-\left(\xi_{3}-\xi_{4}\right)\right]\left|\xi_{2}\right|\right. \\
& +\mu(t)\left(\frac{1}{48}+\frac{1}{96}+v_{1} \tan \left(\xi_{3}-\xi_{4}\right)+\left(\tan \left(\xi_{3}-\xi_{4}\right)-\left(\xi_{3}-\xi_{4}\right)\right)^{2} \leqslant 0\right.
\end{aligned}
$$

for $\mu(t) \leqslant \frac{16}{225}$ and $\left|\xi_{2}(t)\right| \geqslant \frac{1}{48}, t \geqslant T_{1}$. Therefore, it follows that there exists a finite $T_{2} \geqslant T_{1}$ such that $\left|\xi_{2}(t)\right| \leqslant \frac{1}{48}$ for $t \geqslant T_{2}$. Hence, the updated control input $\nu_{2}$ and the first equation of system (3.21) become

$$
\begin{aligned}
& v_{2}(t)=-\xi_{4}(t)-\xi_{3}(t)-\xi_{2}(t)-S_{\frac{1}{96}}\left(\xi_{1}(t)\right) \\
& \xi_{1}^{\Delta}(t)=-S_{\frac{1}{96}}\left(\xi_{1}(t)\right)+3 v_{1} \tan \left(\xi_{3}-\xi_{4}\right)(t)+3\left[\tan \left(\xi_{3}-\xi_{4}\right)(t)-\left(\xi_{3}-\xi_{4}\right)(t)\right] .
\end{aligned}
$$

Similarly, define a Lyapunov function $V_{1}\left(t, \xi_{1}\right)=\xi_{1}^{2}(t)$. Then taking the derivative of $V_{1}$, we have

$$
\dot{V}_{1}\left(t, \xi_{1}\right)=\xi_{1}^{\Delta}(t)\left(2 \xi_{1}(t)+\mu(t) \xi_{1}^{\Delta}(t)\right) \leqslant 0
$$

for all $\left|\xi_{1}(t)\right| \geqslant \frac{1}{96}, t \geqslant T_{2}$, i.e., $V_{1}$ is negative semi-definite. Therefore, it follows that there exists a finite $T_{3} \geqslant T_{2}$ such that $\left|\xi_{1}(t)\right| \leqslant \frac{1}{96}$ for $\mu(t) \leqslant \frac{16}{225}, t \geqslant T_{3}$. Therefore, we have proved that $\left|\xi_{4}(t)\right| \leqslant \frac{1}{12},\left|\xi_{3}(t)\right| \leqslant$ $\frac{1}{24},\left|\xi_{2}(t)\right| \leqslant \frac{1}{48}$, and $\left|\xi_{1}(t)\right| \leqslant \frac{1}{96}$ for $t \geqslant T_{3}$, i.e., solutions of system (3.21) enter the domain $X$ for $t \geqslant T_{3}$. Thus, the control input turns into

$$
v_{2}(t)=-\xi_{4}(t)-\xi_{3}(t)-\xi_{2}(t)-\xi_{1}(t)
$$

so that the overall system (3.21) turns out to be

$$
\left\{\begin{array}{l}
\xi_{1}^{\Delta}(t)=-\xi_{1}+3 v_{1} \tan \left(\xi_{3}-\xi_{4}\right)+3\left(\tan \left(\xi_{3}-\xi_{4}\right)-\left(\xi_{3}-\xi_{4}\right)\right), \\
\xi_{2}^{\Delta}(t)=-\xi_{1}-\xi_{2}+v_{1} \tan \left(\xi_{3}-\xi_{4}\right)+\left(\tan \left(\xi_{3}-\xi_{4}\right)-\left(\xi_{3}-\xi_{4}\right)\right), \\
\xi_{3}^{\Delta}(t)=-\xi_{1}-\xi_{2}-\xi_{3} \\
\xi_{4}^{\Delta}(t)=-\xi_{1}-\xi_{2}-\xi_{3}-\xi_{4},
\end{array}\right.
$$

for $\mu(t) \leqslant \frac{16}{225}, t \geqslant T_{3}$. So the proof of claim 1 follows.

Claim 2: System (3.22) is asymptotically stable on $X$.

Proof of Claim 2: By Theorem 3.5, we have that the perturbed system (3.22) is uniformly asymptotically stable. This implies that system (3.21) with $v_{2}(t)=-S_{\frac{1}{12}}\left(\xi_{4}\right)-S_{\frac{1}{24}}\left(\xi_{3}\right)-S_{\frac{1}{48}}\left(\xi_{2}\right)-S_{\frac{1}{96}}\left(\xi_{1}\right)$ is uniformly asymptotically stable for $\mu(t) \leqslant \frac{16}{225}, t \geqslant T_{3}$. Note that (3.21) is a transformed version of (3.20) and system (3.20) is transformed system of our main system (3.15). Therefore, the asymptotic stability of system (3.15) with controller (3.19) follows. 


\section{Simulation results}

In this section, we simulate our main results for time scales $\mathbb{P}_{a, b}$ and in $\mathbb{R}$.

Proposition 4.1. Let $f: \mathbb{T} \rightarrow \mathbb{R}$ and

$$
\mathbb{T}=\mathbb{P}_{a, b}=\bigcup_{k=0}^{\infty}[k(a+b), k(a+b)+a]
$$

with $\mathrm{a}, \mathrm{b}>0$. Then

$$
\begin{aligned}
& \sigma(t)= \begin{cases}\mathrm{t}, & \text { if } \mathrm{t} \in \bigcup_{\mathrm{k}=0}^{\infty}[\mathrm{k}(\mathrm{a}+\mathrm{b}), \mathrm{k}(\mathrm{a}+\mathrm{b})+\mathrm{a}), \\
\mathrm{t}+\mathrm{b}, & \text { if } \mathrm{t} \in \bigcup_{\mathrm{k}=0}^{\infty}\{\mathrm{k}(\mathrm{a}+\mathrm{b})+\mathrm{a}\},\end{cases} \\
& \mu(\mathrm{t})= \begin{cases}0, & \text { if } \mathrm{t} \in \bigcup_{\mathrm{k}=0}^{\infty}[\mathrm{k}(\mathrm{a}+\mathrm{b}), \mathrm{k}(\mathrm{a}+\mathrm{b})+\mathrm{a}), \\
\mathrm{b}, & \text { if } \mathrm{t} \in \bigcup_{\mathrm{k}=0}^{\infty}\{\mathrm{k}(\mathrm{a}+\mathrm{b})+\mathrm{a}\},\end{cases}
\end{aligned}
$$

and the derivative of $\mathrm{f}$ is defined by

$$
f^{\Delta}(t)= \begin{cases}\lim _{s \rightarrow t} \frac{f(s)-f(t)}{s-t}, & \text { if } t \in \bigcup_{k=0}^{\infty}[k(a+b), k(a+b)+a), \\ \frac{f(\sigma(t))-f(t)}{\mu(t)}, & \text { if } t \in \bigcup_{k=0}^{\infty}\{k(a+b)+a\} .\end{cases}
$$

Example 4.2. Consider system (1.1) with $\mathbb{T}=\mathbb{P}_{a, b}$ for $a=0.01$ and $b=0.07$. The initial conditions are $x_{1}=12, y_{1}=13$ and $x_{2}=y_{2}=\theta_{1}=\theta_{2}=0.1$. The constants in $v_{1}$ are $c_{1}=10$ and $c_{2}=15$.
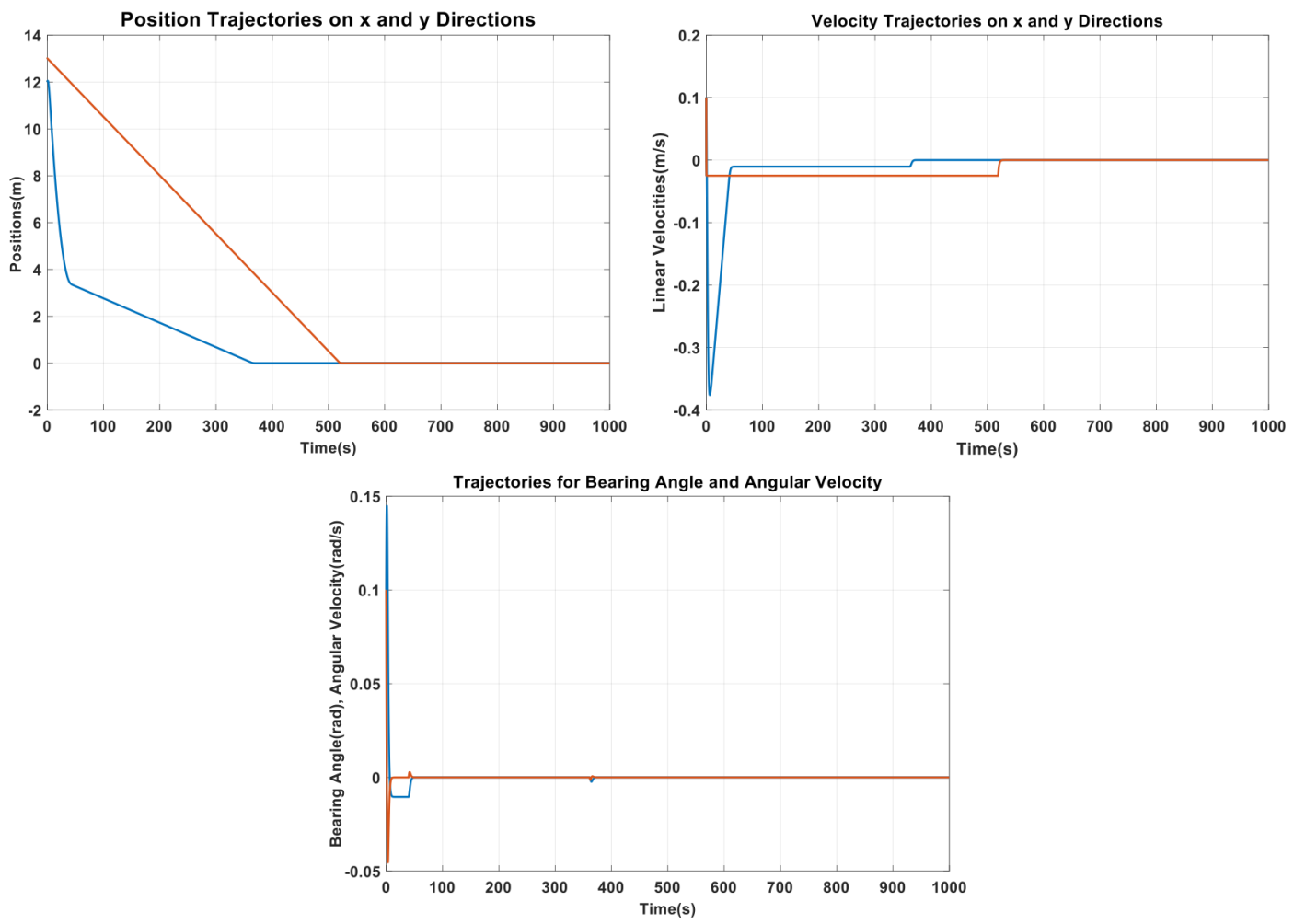

The next example shows us how the control inputs $v_{1}$ and $u_{2}$ change over time in different time scales.

Example 4.3. We consider the same parameters as in Example 4.2 with $\mathbb{T}=\mathbb{P}_{a, b}$, and $\mathbb{T}=\mathbb{R}$. The first 3 figures are the controller signals $\mathfrak{u}_{2}$ and $v_{1}$ when $\mathbb{T}=\mathbb{P}_{a, b}$. The first and the second pictures in Figure 2 show the zoomed version of the third picture in Figure 2. Finally, the following figure shows how the controller changes over time when $\mathbb{T}=\mathbb{R}$. Similarly, the first and second pictures in Figure 3 show the zoomed version of the last picture in Figure 3. 


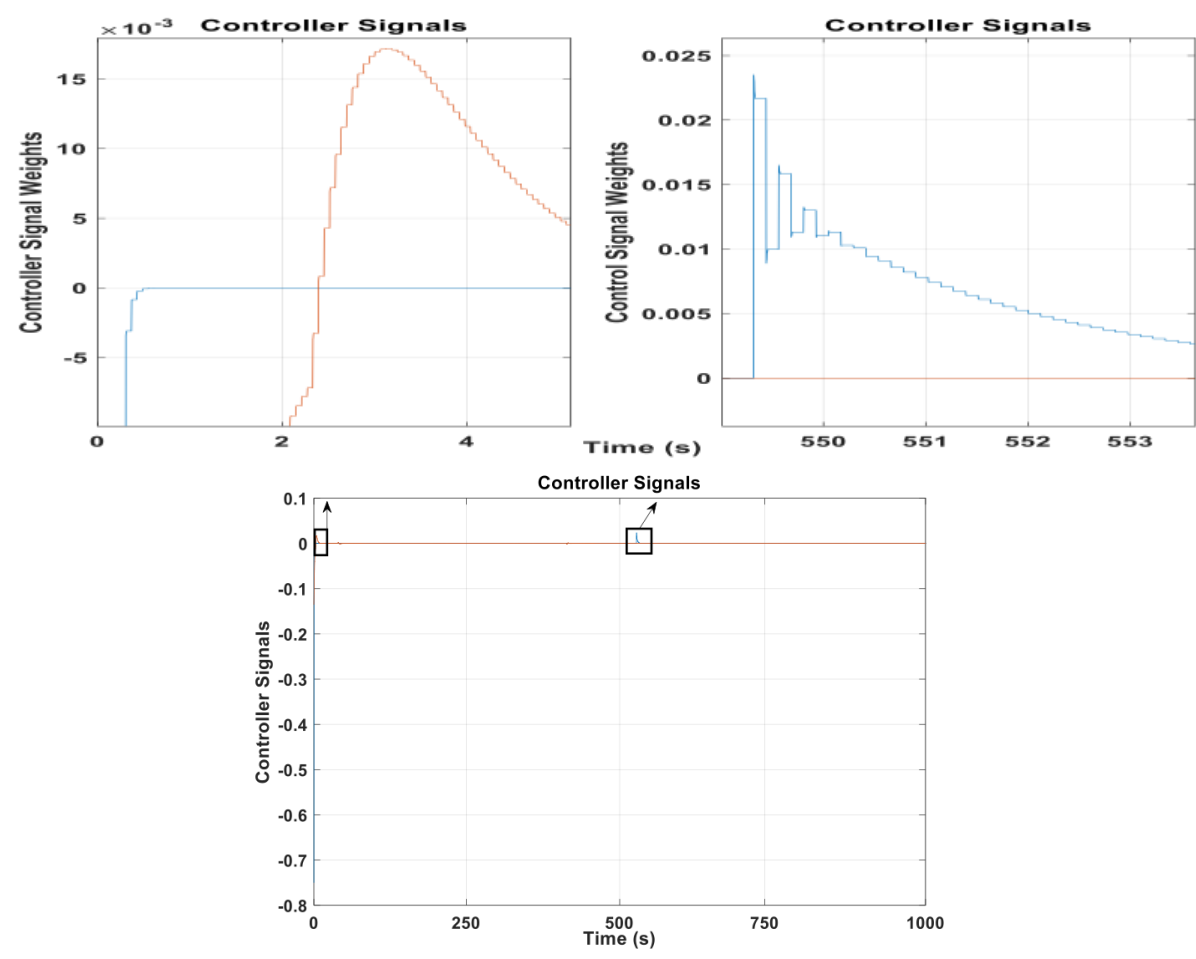

Figure 2: Controller signals when $\mathbb{T}=\mathbb{P}_{\mathrm{a}, \mathrm{b}}$.

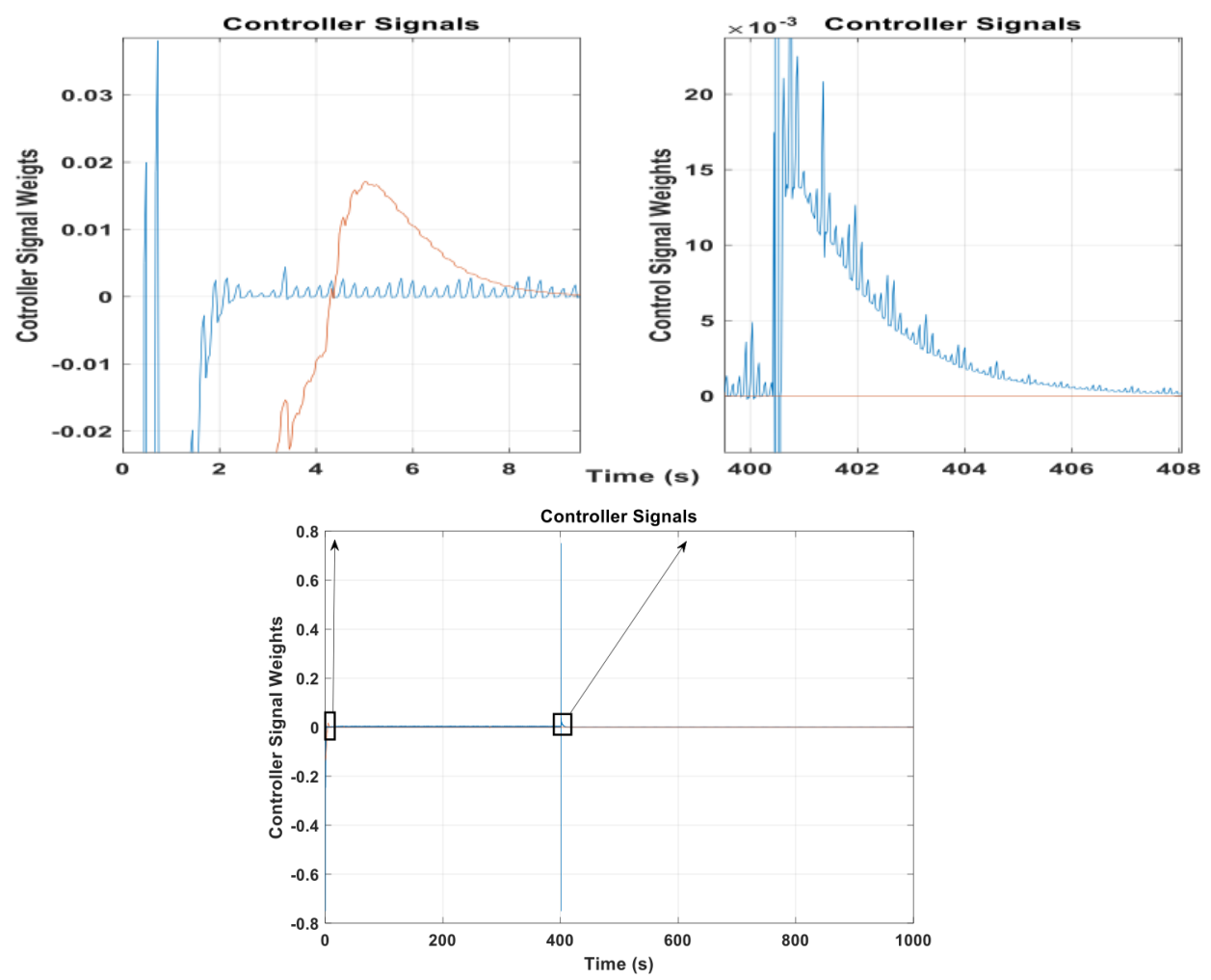

Figure 3: Controller signals when $\mathbb{T}=\mathbb{R}$. 


\section{Conclusion}

This paper deals with the control of PVTOL aircraft on time scales with bounded graininess $\mu$ and $\sup \mathbb{T}=\infty$. We have used the saturation function control tool to control such a system and have successful results for the asymptotic stability. Another observation is how the controllers react when we choose different time scales as in Example 4.3. Note that when we compare the continuous time controller signal figures with $\mathbb{P}_{a, b}$ counterparts, it is obvious that there is much more chattering in continuous time than $\mathbb{P}_{a, b}$. Having too much chattering in control signal is not good for real time applications because it harms the actuators such as dc motor or the gears. Therefore, the chattering which is the main drawback of the saturation based controllers are fixed by designing the controller in $\mathbb{P}_{a, b}$ time scale.

\section{Acknowledgment}

This work was supported by the Scientific and Technological Research Council of Turkey (TUBITAK), Project No. 217E138.

\section{References}

[1] E. Akın, H. Güzey, Ö. Öztürk, Control of Wheeled Mobile Robots on Time Scales, Differ. Equ. Dyn. Syst., 2019 (2019), 10 pages. 1

[2] B. Ben Nasser, K. Boukerrioua, M. A. Hammami, On Stability and Stabilization of Perturbed Time Scale Systems with Gronwall Inequalities, Zh. Mat. Fiz. Anal. Geom., 11 (2015), 207-235. 2.2, 3.4

[3] M. Bohner, A. Peterson, Dynamic equations on time scales: an introduction with applications, Birkhäuser, Boston, (2001). 1, 2.1, 2.3, 2.4, 2.5, 2.6, 2.9, 2.1, 2.11

[4] M. Bohner, A. Peterson, Advances in dynamics equations on time scales, Birkhäuser, Boston, (2003). 1

[5] E. Braverman, B. Karpuz, Uniform Exponential Stability of First Order Dynamic Equations with Several Delays, Appl. Math. Comput., 218 (2012), 10468-10485. 2.2

[6] T. S. Doan, A. Kalauch, S. Siegmund, Exponential Stability of Linear Time-Invariant Systems on Time Scales, Nonlinear Dyn. Syst. Theory, 9 (2009), 37-50. 2.2

[7] N. H. Du, L. H. Tien, On the exponential stability of dynamic equations on time scales, J. Math. Anal. Appl., 331 (2007), 1159-1174. 2.2

[8] I. Fantoni, R. Lozano, Non-linear Control for Underactuated Mechanical Systems, Springer-Verlag, London, (2002). 1

[9] J. Hauser, S. Sastry, G. Meyer, Nonlinear control design for slightly nonminimum phase systems: Application to V/STOL aircraft, Automatica J. IFAC, 28 (1992), 665-679. 1

[10] S. Hilger, Ein Maßkettenkalkül mit Anwendung auf Zentrumsmannigfaltigkeiten, Ph. D. Thesis, Universität Würzburg, (1988). 1

[11] J. Hoffacker, C. C. Tisdell, Stability and Instability for Dynamic Equations on Time Scales, Comput. Math. Appl., 49 (2005), 1327-1334. 2.2, 2.16

[12] H. K. Khalil, Nonlinear Systems, Prentice-Hall, Upper Saddle River, (2002). 2.13

[13] F. Lin, W. Zhang, R. D. Brandt, Robust hovering control of a PVTOL aircraft, IEEE Trans. Control Syst. Tech., 7 (1999), 343-351. 1

[14] P. Martin, S. Devasia, B. Paden, A different look at output tracking: Control of a VTOL aircraft, Automatica, 32 (1996), 101-107. 1

[15] A. A. Martynyuk, Stability Theory for Dynamic Equations on Time Scales, Birkhäuser/Springer, Basel, (2016). 2.2, 3.1

[16] R. M. Murray, Z. Li, S. S. Shankar, A Mathematical Introduction to Robotic Manipulation, CRC Press, Boca Raton, (1994). 2.13

[17] Ö. Öztürk, On the existence of the nonoscillatory solutions of three-dimensional time scale systems, J. Fixed Point Theory Appl., 19 (2017), 2617-2628. 1

[18] Ö. Öztürk, E. Akin, Classification of Nonoscillatory Solutions of Nonlinear Dynamic Equations on Time Scales, Dynam. Systems Appl., 25 (2016), 219-235.

[19] Ö. Öztürk, H. M. Güzey, Optimal Control of Quadrotor Unmanned Aerial Vehicles on Time scales, Int. J. Difference Equ., 13 (2018), 41-54. 1

[20] A. C. Peterson, Y. N. Raffoul, Exponential Stability of Dynamic Equations on Time Scales, Adv. Difference Equ., 2005 (2005), 133-144. 2.2 